\title{
Detecting and Discriminating the Direction of Motion of Luminance and Colour Gratings
}

\author{
A. M. DERRINGTON,* G. B. HENNING $\dagger$ \\ Received 19 February 1992; in revised form 17 August 1992
}

\begin{abstract}
Human observers were required to discriminate the direction of motion of vertically moving, $1 \mathrm{c} / \mathrm{deg}$ luminance and colour gratings. The gratings had different contrasts and moved at temporal frequencies between 0.5 and $32 \mathrm{~Hz}$. Sensitivity [the reciprocal of the contrast at which performance reached $75 \%$ correct in a temporal two-alternative forced-choice (2 AFC) discrimination task] was a band-pass function of temporal frequency for luminance gratings, and a low-pass function of temporal frequency for colour gratings. Further, when colour contrast was expressed in terms of the modulation in cone excitation produced by the stimulus, sensitivity to colour gratings was greater than sensitivity to luminance gratings at frequencies below $2 \mathrm{~Hz}$. On the other hand, at temporal frequencies above $4 \mathrm{~Hz}$, sensitivity to colour gratings was comparable with sensitivity to luminance gratings of double the temporal frequency. Detection sensitivity was measured for luminance and colour gratings of 1,4 and $16 \mathrm{~Hz}$. With either colour or luminance gratings, detection thresholds were very similar to those for direction-of-motion discrimination. This result confirms findings of Mullen and Boulton [(1992) Vision Research, 32, 483-488] and Cavanagh and Anstis [(1991) Vision Research, 31, 2109 2148], but is different from that reported by Lindsey and Teller [(1990) Vision Research, 30, 1751-1761] who used a smaller stimulus seen in a parafoveal region and found that motion discrimination thresholds were higher than detection threshold for colour gratings. We repeated our threshold measurements using parafoveal viewing conditions similar to those used by Lindsey and Teller (1990). We found that, although for luminance gratings detection thresholds were very close to direction-discrimination thresholds, for colour gratings, they were lower. The result is in qualitative agreement with Lindsey and Teller (1990). Our results suggest that low-level, or "first-order" motion mechanisms are not as sensitive to chromatic gratings as are colour-detection mechanisms.
\end{abstract}

Motion Luminance Colour Isoluminance Equiluminance

\section{INTRODUCTION}

\section{Contribution of chromatic mechanisms to motion sensitivity}

There is a long-standing controversy about our sensitivity to the motion of patterns which consist of spatial variations in chromaticity without variations in luminance. Much of the debate has been based on subjective observations about the appearance of motion in such patterns (Cavanagh \& Anstis, 1991; Cavanagh, Tyler \& Favreau, 1984; Krauskopf \& Farell, 1990; Ramachandran \& Gregory, 1978), and about perceptual aftereffects (Derrington \& Badcock, 1985a; Mullen \& Baker, 1985). However the issues have also been addressed using psychophysical discrimination measurements (Lindsey \& Teller, 1990; Troscianko \& Fahle, 1988).

Ramachandran and Gregory (1978) studied the impression of movement that occurs when part of a

\footnotetext{
*Department of Physiological Sciences, The Medical School, Framlington Place, Newcastle upon Tyne NE2 4HH, England. $†$ Department of Experimental Psychology, The University of Oxford, South Parks Road, Oxford OX1 3UD, England.
}

random chequerboard pattern is made to jump backwards and forwards about 4 times per second. They found that, in a pattern made of red and green squares, the impression of motion disappeared when the pattern was made equiluminant (i.e. when the red and green squares were adjusted to be of equal luminance); even though the coloured squares were clearly visible, they did not give rise to an impression of motion unless there was a luminance difference between the red and green squares. Furthermore, the adjustment of luminance values necessary to abolish the impression of motion was less critical when the squares in the random chequerboard were different colours that when they were all the same colour, suggesting that colour makes the perception of motion more difficult. Ramachandran and Gregory (1978) concluded that "colour and motion are handled separately by the human visual system and that colour provides only a weak "cue" at best to motion perception".

More recently it has been shown that moving colour patterns elicit percepts very similar to those elicited by moving luminance patterns, although the impression of motion they elicit may not be so robust: the apparent speed of moving colour gratings is lower than that of 
luminance gratings (Cavanagh et al., 1984), even to the point that adding colour to a luminance grating reduces its apparent speed of motion. However, prolonged viewing of equiluminant coloured gratings induces a normal motion-after-effect (MAE) in the form of an impression of motion in the opposite direction to that of the pattern wich has been viewed (Cavanagh \& Favreau, 1985; Derrington \& Badcock, 1985a; Mullen \& Baker, 1985). Further, motion after-effects induced by equiluminant coloured gratings transfer to luminance gratings, and can be nulled by motion of the luminance grating in the opposite direction. Similarly, equiluminant gratings can be used to null the after-effect induced by a luminance grating, although colour gratings appear to be less effective both at eliciting and at nulling after-effects than are luminance gratings (Derrington \& Badcock, 1985a).

Another way of comparing the motion impression elicited by luminance gratings and colour gratings is to superimpose patterns moving in opposite directions and find the relative contrasts at which the direction of motion switches from being that of the luminance pattern to that of the colour pattern (Cavanagh \& Anstis, 1991). Interpretation of these results depends on how one chooses to scale the contrast of the chromatic grating relative to the luminance grating. Adopting the simplest method, which is simply to assign to the chromatic grating the contrast of the differently coloured luminance gratings that are added in antiphase in order to form it, one finds that a $100 \%$ modulated chromatic grating is balanced by a luminance grating of about $10 \%$, suggesting that chromatic motion signals are weaker than luminance signals. However, if one chooses to scale each grating by its contrast in relation to the contrast required to permit the discrimination of direction of motion, the two types of pattern are more nearly equal: a chromatic grating of contrast 60 times threshold balances a luminance grating of contrast 30 times threshold. However, it is arguably best to compare chromatic and luminance signals by equating them in terms of the modulation in cone excitation they produce (Stromeyer, Cole \& Kronauer, 1987; Lennie \& D'Zmura, 1988). When the results of Cavanagh and Anstis (1991) are scaled in this way one finds that a chromatic grating which modulates $\mathrm{R}$ and $\mathrm{G}$ cone outputs by about $5 \%$ balances a luminance grating that modulates them by $10 \%$, suggesting that chromatic motion signals may be more effective than luminance motion signals. Similarly, Stromeyer et al. (1990) found that motion of colour gratings can be discriminated at lower cone modulations than motion of luminance gratings.

Troscianko and Fahle (1988) compared the reaction times for detecting the onset and offset of motion in luminance and chromatic stimuli. They found that subjects took longer to respond to the onset or offset of the motion of an equiluminant stimulus, and that the effect could be reproduced using a luminance stimulus that was subjected to a random spatial jitter, or that had reduced contrast. However, these experiments also suffer from the problem that it is not clear how to equate the chromatic and the luminance contrast, although in this case the modulation in cone excitation produced by the yellow-green stimulus used by Troscianko and Fahle (1988) was quite high: the contrast of their moving bar [calculated as (foreground excitation-background excitation)/background excitation] is -0.11 for the red cones, and 0.28 for the green cones.

Another way of addressing the issue of whether chromatic mechanisms provide an input to motion perception is to compare the contrast thresholds for detecting moving chromatic patterns with the thresholds for discriminating their direction of motion. Lindsey and Teller (1990) found that chromatic gratings needed to be at least 3 times their threshold contrast in order for their direction of motion to be discriminated, whereas the direction of motion of luminance patterns could be discriminated close to their detection threshold. Orientation could be discriminated at threshold both for luminance and colour gratings. Lindsey and Teller (1990) using results from an objective paradigm, [twoalternative forced-choice ( $\mathrm{AFC}$ ), method of constant stimuli] conclude that chromatic mechanisms provide input to the mechanisms analysing spatial form (orientation) but not to those analysing motion. These results are contradicted by Cavanagh and Anstis (1991) who found, using a subjective paradigm, that the ratios of detection thresholds to direction-of-motion discrimination thresholds were comparable for colour and luminance stimuli. Mullen and Boulton (1992) also measured thresholds for direction-of-motion discrimination and for detection, using a single 2 AFC detection task in which subjects were also required to discriminate the direction of motion of the stimulus. They found a small but consistent advantage of detection over discrimination.

However, when subjects are required to do two tasks simultaneously, there is always the possibility that the relationship between the thresholds obtained is determined by the way the subjects divide their attention between the two tasks. It therefore seems important to measure thresholds for detection and direction-ofmotion discrimination separately, both for colour and for luminance gratings, to see whether this is the source of the difference between Lindsey and Teller (1990) on the one hand and Cavanagh and Anstis (1991) and Mullen and Boulton (1992) on the other. Another potentially important factor in Lindsey and Teller's (1990) experiments is that their stimulus conditions (parafoveal presentation, small display field, $4 \mathrm{~Hz}$ temporal frequency) are not optimal for chromatic mechanisms. For this reason we decided to make our measurements of sensitivity to red-green equiluminant colour gratings using 2 AFC detection and direction-ofmotion discrimination tasks, with a large display, viewed in central vision, sampling a wide range of temporal frequencies with 100 trials per data point, and comparing the results with those obtained using luminance gratings. We then compare these results with measurements made using a smaller parafoveal display like that used by Lindsey and Teller (1990). 


\section{METHODS}

\section{Stimuli and observers}

Three observers (including the authors) served in 2 AFC direction-of-motion discrimination and detection experiments. The stimuli were horizontally orientated, sinusoidal gratings viewed binocularly with suitably corrected vision, and had either luminance or colour variation. They were generated by the method of Schade (1956) using a one-dimensional display controller (Cambridge Research Systems VSG2/1) with three 14-bit digital-to-analogue converters (DACs) and displayed on a Barco CDCT6551 colour monitor. At $1.37 \mathrm{~m}$, the display subtended 12.1 deg of visual angle horizontally, and 10.0 deg vertically, at the observers' eyes. Except where otherwise specified, the grating stimuli occupied the whole illuminated area of the display $(12.1 \times 10 \mathrm{deg})$. The unmodulated screen produced a uniform grey field of luminance $44.2 \mathrm{~cd} \mathrm{~m}^{-2}$ (CIE chromaticity coordinates: $x=0.333, y=0.477$ ) and neither the mean luminance nor the mean chromaticity of the display was altered by the presentation of the gratings.

The gratings, $1 \mathrm{c} / \mathrm{deg}$ luminance or colour gratings, were produced by modulating the luminances of the phosphors of the colour television display as follows:

$$
\begin{aligned}
L(y)=L_{\mathrm{m}} & (r, g, b) \\
& +M(r, g, b) \cos [2 \pi(f y-g t)+\phi] W(t),
\end{aligned}
$$

where $f$ is the spatial frequency (c/deg), and $g$ the temporal frequency $(\mathrm{Hz})$ of the grating, and $\phi$ is a phase term. The mean luminance, $L_{\mathrm{m}}$, is produced by summing the contributions from the $r, g$, and $b$ phosphors, $L_{\mathrm{m}}(r)$, $L_{\mathrm{m}}(g)$, and $L_{\mathrm{m}}(b)$, in the proportions $0.208,0.661$, and 0.131 respectively. $M(r, g, b)$ represents the luminance modulation of the three phosphors of the display. $W(t)$ is a raised-cosine temporal envelope in which

$$
W(t)= \begin{cases}1+\cos 2 \pi(t-0.5), & 0<t<1 \\ 0 & \text { otherwise. }\end{cases}
$$

A luminance grating of a given contrast was produced by setting the modulation of each phosphor to be the product of the required contrast, $C$, and the contribution of that phosphor, $p$, to the mean luminance, $L_{\mathrm{m}}(p)$. Thus, for each phosphor in a luminance grating,

$$
L_{p}(y)=L_{\mathrm{m}}(p)\{1+C \cos [2 \pi(f y+g t)+\phi]\} W(t) .
$$

Equation (2) defines a grating of contrast $C$ on each phosphor. Since, in a luminance grating, all the gratings for the phosphors have the same spatial and temporal frequencies, and the same contrast and phase, the relative contributions of the three phosphors are unchanged by the modulation and only luminance variations result.

The colour gratings were produced by modulating the luminance of only the $r$ and $g$ phosphors in antiphase (with modulations of equal luminance) so that

\footnotetext{
"In fact there is a small deviation from the "constant blue" axis of Derrington et al.: our chromatic gratings modulated the blue cone quantum catch by about one-tenth the amount they modulated the quantum catch of the other two cone types.
}

$$
M(r)=-M(g)
$$

and

$$
M(b)=0 .
$$

This produced spatial variations in chromaticity but not luminance. The chromaticity variation was along an axis close to the "constant blue" axis of Derrington et al. (1984). ${ }^{*}$

Following a suggestion of Lennie and D'Zmura (1988), we took the following steps in order to express the magnitude of the variation of our chromatic stimuli in units that have some meaning outside the context of our particular display or colour representation. First we used the equations of Smith and Pokorny (1975) to calculate the relative quantum catches in the $R$ and $G$ cones from the luminances and $\mathrm{CIE}$ chromaticity coordinates of the display phosphors which in turn allows us to calculate the modulations in the quantum catches of the $\mathrm{R}$ and $\mathrm{G}$ cones produced by a red-green equiluminant grating of unit magnitude. We then calculate "contrast sensitivities" in the normal way, expressing the stimulus contrast as the mean of the unsigned modulations in quantum catch of the $\mathrm{R}$ and the $\mathrm{G}$ cones. Note that this scaling produces the normal (Michelson) contrast for luminance gratings and a suitable scale for comparing the detection and discrimination results obtained with luminance and colour gratings.

Photometry: checks on equiluminance. The display luminance calibrations were carried out using a UDT model 61 photometer with a photometric filter and lumi-lens. However because equiluminance planes vary slightly from observer to observer, and because the photometer deviates slightly from $V \lambda$ spectral sensitivity, we determined equiluminance points for each observer at different temporal rates. A $1 \mathrm{c} / \mathrm{deg}$ "colour" grating was presented under the same conditions as all our stimuli. The grating was presented as a sinusoidally counterphase flickering grating at flicker rates of 4,8 or $16 \mathrm{~Hz}$. The observers had levers which allowed them to add a luminance grating to the coloured grating and to adjust its contrast and polarity until the perceived flicker was minimized. At this point we assume that the added luminance grating cancels any residual luminance in the chromatic grating introduced by variations in the equiluminance plane, or by chromatic aberration, and in all experiments using that chromatic grating for that observer the appropriate proportion of luminance contrast was added to it in the appropriate phase. The mean of six settings under each condition determined the appropriate contrast for the luminance grating. The cancellation task was too difficult (or too variable in its results) at lower temporal frequencies so we used the value measured at $4 \mathrm{~Hz}$ for that temporal frequency or lower.

Temporal resolution of display. Two anonymous referees suggested that the chromatic balance of our stimuli might vary with temporal frequency. We checked the temporal properties of the phosphors by measuring the light output from a circular patch approx. $0.5 \mathrm{deg}$ in diameter using a Gamma Scientific Instruments model 2400 photometer. Although there were substantial 
differences in persistence, the light output of the slowest phosphor (the red) decayed to $<95 \%$ of its peak within $1 \mathrm{msec}$ and thus differences in phosphor persistence are irrelevant at the temporal frequencies we use.

Spatial inhomogeneities. The display screen contained no obvious spatial inhomogeneities. The spatial variations visible in the appearance of equiluminant patterns appeared to move with changes in fixation. Consequently we presume that they are consequences of the inhomogeneities in the visual field, rather than in the display.

Quantization errors in stimulus generation. The luminances of the red, green and blue phosphors were modulated using 14-bit DACs, but memory limitations in the display controller made it necessary to sample the luminance waveforms more coarsely than this. The sampling was determined by the lengths of the lookup tables which were used to compensate for the nonlinear relationship between applied voltage and luminance in the display. Separate lookup tables were maintained for each colour. Successive entries in each table contained numbers which, when sent to the DAC, produced luminances differing by a constant increment. The size of the increment was the same for each phosphor, and so, since the different phosphors have different luminous efficiencies, each table contained a different number of brightness values. The green table contained 4096, the red table contained about 1300, and the blue table contained about 800 . The luminance step was defined as 1/4096 of the luminance range of the green gun, so, inevitably, the exact number of steps in the blue and red tables changed slightly each time the display was recalibrated.

Quantization of luminance gratings. The luminance and chromaticity of the unmodulated display was produced by 2048 units of green, 646 units of red and 405 units of blue; these particular values were chosen by first setting each gun to half its maximum brightness, and then altering the red and blue guns to produce a satisfactory "white" appearance. To produce the luminance gratings to which observers were most sensitive (contrasts of the order of 0.001 ) required a peak to peak modulation of only about four luminance steps on the green gun, and proportionally fewer on the other guns. This means that these gratings were not exactly sinusoidal and were slightly reduced in amplitude, because the waveform was relatively coarsely quantized (Pelli \& Zhang, 1991); nor were they completely homochromatic, because quantization affects the red and blue guns more severely than the green gun. However, we took care to select stimulus contrasts that minimized these effects by choosing settings just above the "break points" at which deteriorations occurred, and in fact we were unable to detect either alterations in the appearance of the stimuli, or irregularities in the data produced by quantization.

Quantization errors in colour gratings. The expected effect of quantization on colour gratings is much less severe because the modulations required to produce them are much larger than in the case of luminance gratings, and because the modulations of the red and green guns were equal in magnitude. The only significant effect of quantization of the chromatic gratings is that the luminance grating added to compensate for individual differences in equiluminance settings was itself quantized as described above. However, this error is likely to be extremely small.

\section{Direction-of-motion discrimination}

A 2 AFC task was used with two observation intervals on each trial. Each interval was $1 \mathrm{sec}$ long, and was defined for the observers by a burst of audible noise, and the intervals were separated by a pause of approx. $250 \mathrm{msec}$. In one interval, a $1 \mathrm{c} / \mathrm{deg}$ grating was presented moving upwards, and in the other interval the same grating was presented moving downwards at the same speed. The gratings were turned on and off by the raised cosine temporal envelope, $W(t)$ [equation (1)] The grating in the first interval was as likely to be moving upwards as downwards on each trial, and the observers' task was to indicate the interval in which the grating moved upwards. Five different contrasts were used, randomly chosen from trial to trial subject to the constraint that no contrast was used for the $n$th time until all had been used $n-1$ times. The session lasted until each stimulus had been used on 50 trials. This process was repeated twice to give five points on the function relating the percentage of correct responses to contrast for each observer, with each point based on 100 trials. Separate functions were collected in separate experiments for colour and luminance gratings, and for speeds ranging from 0.5 to $32 \mathrm{deg} / \mathrm{sec}$.

\section{Detection of moving gratings}

The 2 AFC detection experiments were identical to the discrimination experiments save that a moving grating was presented in one interval and only the steady, uniform, mean luminance of the display in the other interval. The observers were required to choose the interval in which the moving grating had been presented. On half the trials the moving grating moved upwards, and the results for upwards and downwards motion were combined.

\section{RESULTS AND DISCUSSION}

\section{Direction-of-motion discrimination}

Figure 1 shows the performance of three observers in direction-of-motion discrimination tasks using luminance gratings and colour gratings, at two different temporal frequencies, plotted as functions of contrast. Results for each observer are indicated by the same shaped symbols throughout.

Figure 1(a) shows results at $8 \mathrm{~Hz}$. The functions for luminance gratings (solid symbols) are all very similar, and lie approximately a factor or two below those for colour gratings (open symbols), indicating that the observers are more sensitive to luminance than to colour gratings at this temporal frequency. The contrast scale represents the mean of the absolute modulations in cone quantum catch for $\mathbf{R}$ and $\mathbf{G}$ cones, and so represents a realistic, but not unique, metric for comparing the 
(a)
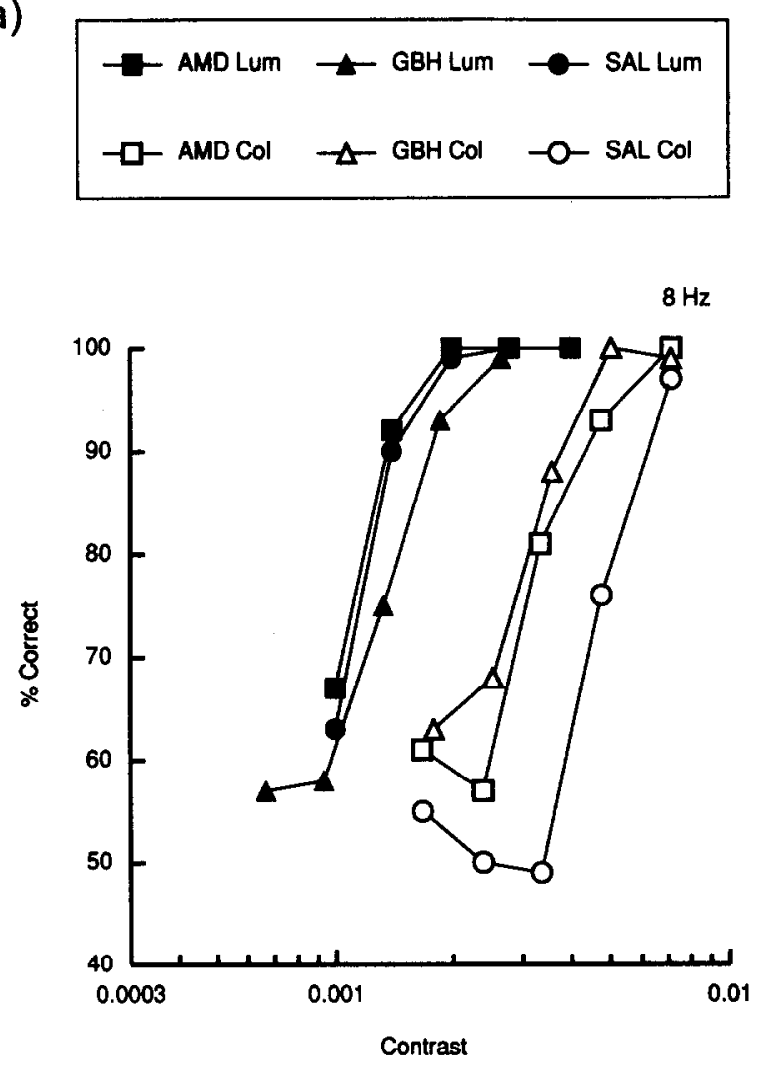

(b)

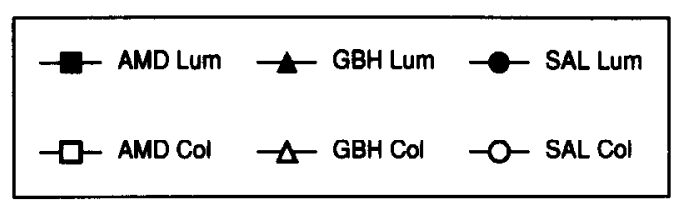

$0.5 \mathrm{~Hz}$

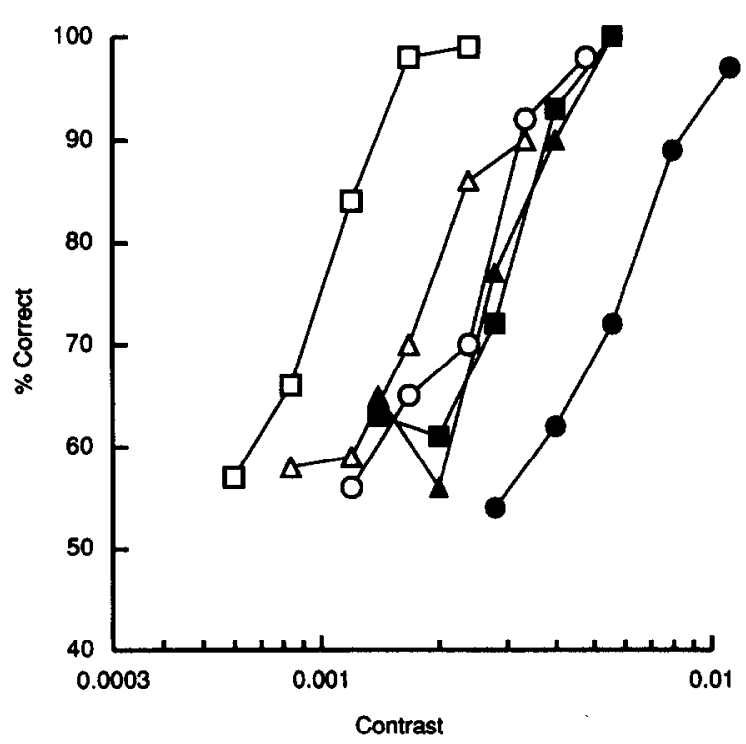

FIGURE 1. (a) The percentage of correct direction-of-motion discrimination judgements as a function of the contrast of luminance (solid symbols) and colour gratings (open symbols) moving at a temporal frequency of $8 \mathrm{~Hz}$. The results for three observers, AMD, GBH and SAL, are shown and each data point is based on 100 trials. (b) The same as (a) but the temporal frequency was $0.5 \mathrm{~Hz}$.

sensitivity to colour gratings with the sensitivity to luminance gratings. The mechanism detecting the luminance gratings would be expected to sum the signals from $R$ and $G$ cones, whereas the mechanism detecting the chromatic gratings would be expected to subtract them (Lennie \& D'Zmura, 1988).

Figure 1(b) shows similar data obtained at $0.5 \mathrm{~Hz}$. At this lower temporal frequency the absolute sensitivities of the individual observers show more variation, but one consistent difference emerges. For each observer the luminance functions are displaced towards higher contrasts, and the chromatic functions are displaced towards lower contrasts. The end result is that, for each observer, performance in discriminating the direction of motion of chromatic gratings is better than performance in discriminating the direction of motion of luminance gratings. This reversal in relative sensitivity to luminance and colour gratings at the lower temporal frequency is analogous to the general finding that chromatic contrast sensitivity tends to be a low-pass function of temporal frequency, whereas luminance contrast sensitivity tends to be a band-pass function of temporal frequency (de Lange, 1958; Varner, Jameson \& Hurvich, 1984).

The functions relating performance to contrast for different kinds of grating, and at different temporal frequencies are all approximately parallel when plotted on semi-logarithmic axes (as in Fig. 1). In fact the functions obtained at $0.5 \mathrm{~Hz}$ [Fig. 1(b)] are shallower than those obtained at any other temporal frequency, whereas those at $8 \mathrm{~Hz}$ [Fig. 1(a)] are typical. Because the slopes of all the psychometric functions are so similar, we used the reciprocal of the contrast corresponding to $75 \%$ correct discrimination to compare sensitivity over a wide range of temporal frequencies.

The direction-of-motion sensitivities (the reciprocal of the contrast corresponding to $75 \%$ correct directionof-motion discrimination) of three observers are plotted as functions of temporal frequency in Fig. 2(a-c) each observer's data being shown in a different figure. The sensitivity functions obtained with luminance gratings have the familiar band-pass sensitivity function seen in contrast sensitivity functions for detection (Robson, 1966). Sensitivity peaks between 2 and $8 \mathrm{~Hz}$, declines sharply at higher frequencies, and gradually at lower frequencies. At the lowest frequency tested $(0.5 \mathrm{~Hz})$ sensitivity falls from its peak by a factor between three and five, depending on the observer. The direction-ofmotion sensitivity functions for the colour gratings are more low-pass in character and are displaced towards lower temporal frequencies. This too is a characteristic found in contrast sensitivity functions for the detection of chromatic temporal modulation (de Lange, 1958; Mullen \& Boulton, 1992; Varner et al., 1984). Sensitivity is greatest at 1 or $2 \mathrm{~Hz}$, and rolls off gradually at first and then more rapidly at higher frequencies; there is little or no decline at low frequencies. The rate of loss of sensitivity at high temporal frequencies is similar for both sorts of grating, and the maximum sensitivities of the two functions are similar: for two of the observers the peak luminance 
(a)

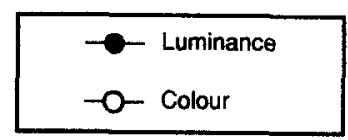

SAL

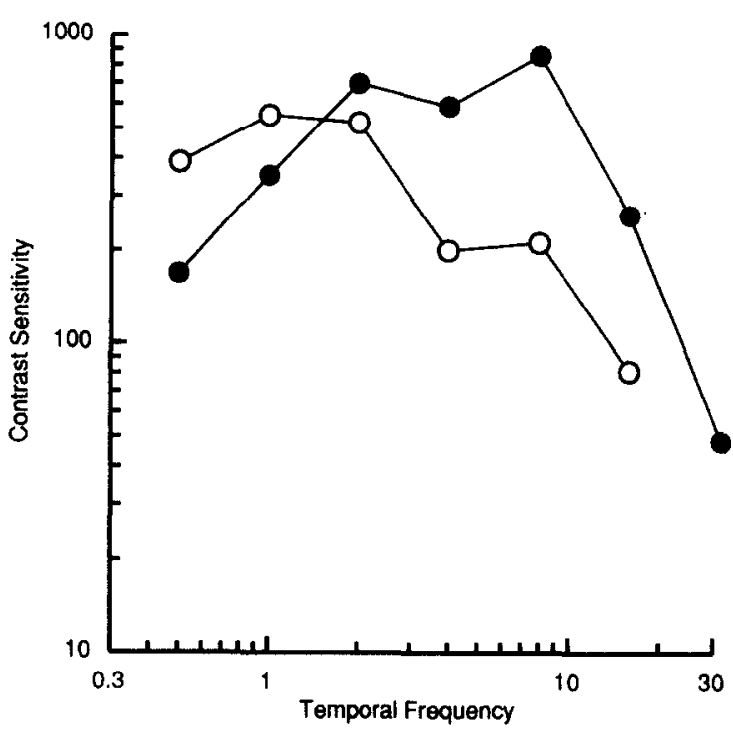

(b)

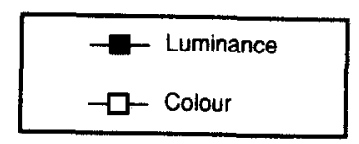

AMD

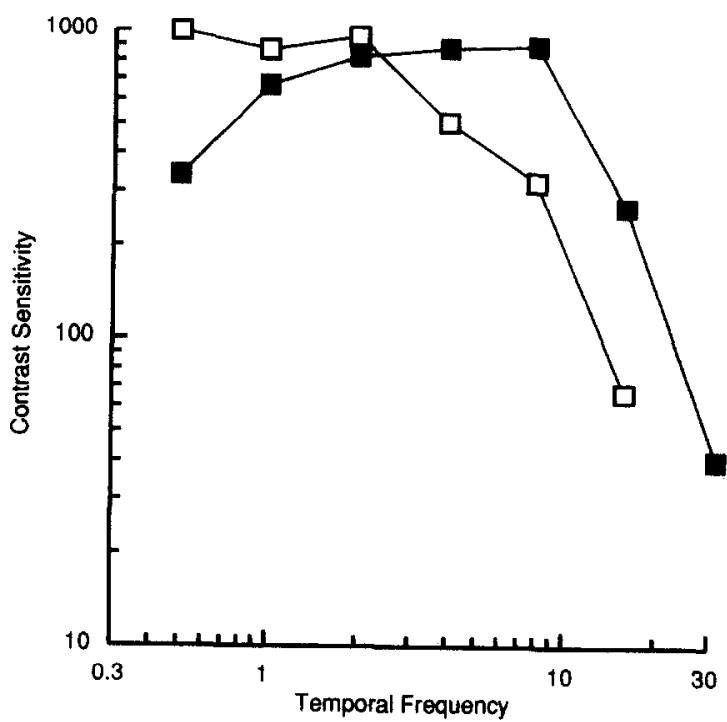

(c)

$\mathrm{GBH}$
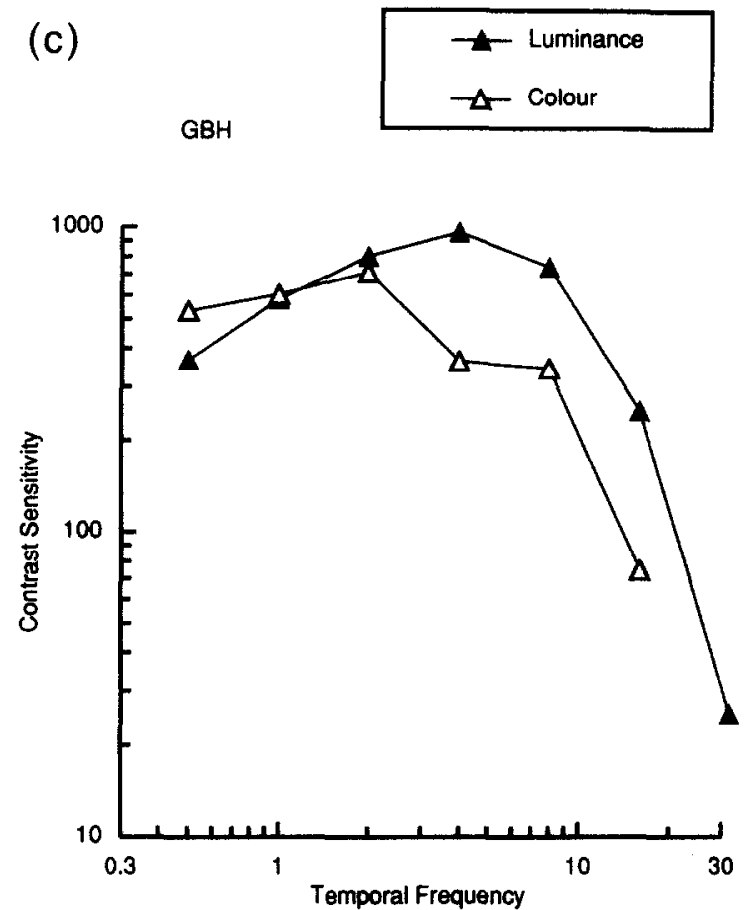

FIGURE 2. (a) Direction-of-motion sensitivity (the reciprocal of the contrast corresponding to $75 \%$ correct direction-ofmotion discrimination) as a function of temporal frequency. Both axes are logarithmic. The solid symbols show the results for luminance gratings, the open symbols show those for colour gratings and data are those of observer SAL. (b) As (a) but the results are those of AMD. (c) As (a) but the results are those of GBH.

sensitivity is slightly higher than the peak chromatic sensitivity, but in no case does the sensitivity differ as much as $0.3 \log$ units. As a consequence, as reported by Stromeyer et al. (1990), direction-of-motion sensitivity with colour gratings is greater than that with luminance gratings at low temporal frequencies but less at high temporal frequencies as Fig. 1 also showed. The superior sensitivity of chromatic motion detection at $2 \mathrm{~Hz}$ is also apparent in the results of Cavanagh and Anstis (1991) if allowance is made for the difference between their contrast metric and cone modulation.
The direction-discrimination sensitivity functions shown in Fig. 2 have the same general form as detection sensitivity functions (Kelly, 1983), and the peak sensitivities are very high, which makes it interesting to see how detection sensitivity compares with directiondiscrimination sensitivity under these conditions. The subjective impressions of our observers in the direction discrimination task led us to expect the two tasks to give very similar sensitivities: observers felt they were unable to see any gratings whose direction of motion they could not discriminate. However, we can hardly be certain that 


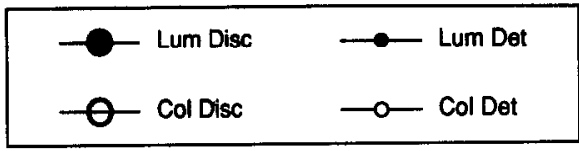

SAL

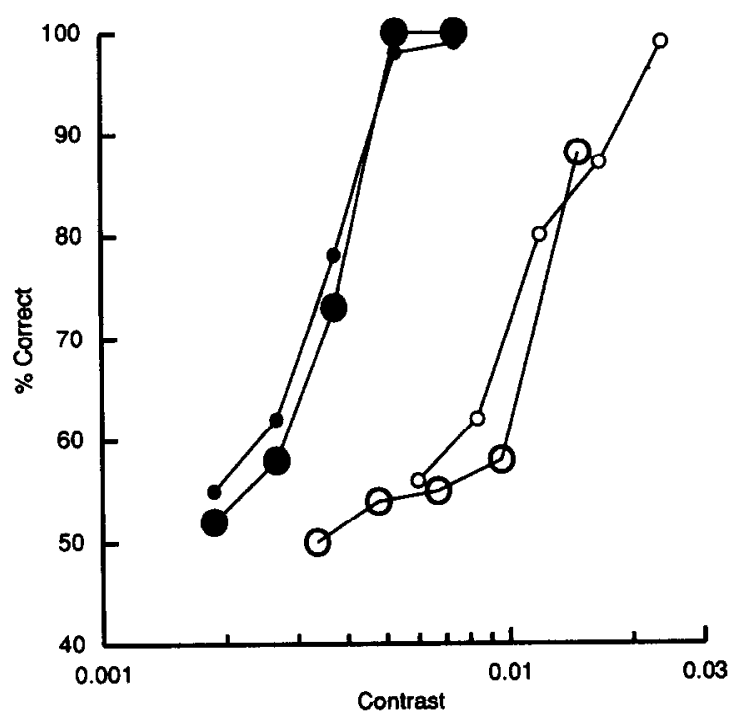

FIGURE 3. The percentage correct direction-of-motion discrimination (large symbols) and the percentage of correct detection (small symbols) plotted as functions of contrast. The solid symbols show the results with luminance gratings and the open symbols, those with colour gratings. All the gratings moved with a temporal frequency of $16 \mathrm{~Hz}$ and each data point is based on 100 trials from observer SAL.

observers' impressions of direction-of-motion were always correct (Derrington \& Henning, 1987). The next section makes a more rigorous comparison of detection and discrimination performance.

\section{Detection of moving gratings}

Figure 3 shows, for one observer, performance in direction discrimination (large symbols) and detection (small symbols), for colour gratings (open symbols) and luminance gratings (solid symbols) moving at $16 \mathrm{~Hz}$. There is no discernible difference in performance between detection and direction of motion discrimination tasks. However, as wc should expect from Fig. 2, performance with luminance gratings at this temporal frequency is substantially better than performance with colour gratings; both the detection and the discrimination psychometric functions for luminance gratings are displaced towards lower contrasts by about $0.5 \mathrm{log}$ units. The slopes of the functions are very similar indeed, and so we again use the contrast at which performance reaches $75 \%$ correct as a basis for comparing detection and discrimination functions obtained with different types of stimulus.

Figure 4 shows the ratio of the contrast at $75 \%$ correct discrimination to the contrast at $75 \%$ correct detection as a function of temporal frequency for colour and for luminance gratings. A ratio above 1.0 indicates that detection occurs at lower contrast than discrimination. In fact, although all ratios are very close to 1.0 , there is a slight tendency for the ratios obtained with luminance gratings to be slightly lower than the ratios obtained with colour gratings. The difference between the detection/discrimination ratios obtained with luminance and colour gratings is much less than that reported by Lindsey and Teller (1990), who found that ratios varied from about 3 to more than 10 with colour gratings, the size of the difference depending on the axis of the chromatic modulation. For a red-green colour grating they found a ratio of about 4 for one observer, and about 6 for the other (their Fig. 6, azimuth $0^{\circ}$ ). The displays used by Lindsey and Teller (1990) were smaller and dimmer than ours, and were presented monocularly and parafoveally. We therefore decided to see whether, by using a smaller patch of grating and presenting it parafoveally, we could obtain results similar to those of Lindsey and Teller (1990).

Figure 5 shows psychometric functions for three observers detecting and discriminating the direction of motion of luminance and colour gratings. The gratings were all of spatial frequency $1 \mathrm{c} / \mathrm{deg}$, and temporal frequency $4 \mathrm{~Hz}$, and were presented with a circular patch of display approx. $2.5^{\circ}$ in diameter, whose right-hand edge was $0.75^{\circ}$ to the left of the fixation point. The remainder of the display was uniformly illuminated at the normal mean luminance and chromaticity. For all three observers the pattern of results is the same. With luminance gratings, data for which are shown by solid symbols, performance in the discrimination task (large symbols) is very slightly better than performance in the detection task (small symbols). As we have already discussed, this is not unexpected on the assumption that the mechanism which signals the presence of the grating is direction selective. With colour gratings (open symbols) the reverse relationship is seen. Performance in the detection task is better than in the discrimination task. The threshold ratio (the separation between the curves at the $75 \%$ correct performance level) for colour gratings varies from slightly less than a factor of 2 (AMD) to slightly less than a factor of 4 (SAL). These data are in qualitative agreement with those of Lindsey and Teller (1990), and indicate that the large difference between detection and direction-of-motion discrimination thresholds which they found reflects an effect either of display size, or of eccentricity.

To test whether the large separation between detection and direction discrimination sensitivities shown in Fig. 5 was caused by reducing display size or by increasing retinal eccentricity, we repeated the measurements using colour gratings. This time the window within which the gratings were presented, still $2.5 \mathrm{deg}$ in diameter, was centred on the fixation point. The psychometric functions for discrimination and detection are shown in Fig. 6. The advantage of detection over discrimination has been substantially reduced. It therefore seems safe to conclude that the large differences in sensitivity between direction-of-motion discrimination and detection described by Lindsey and Teller (1990), and partially replicated in Fig. 5, are a property of the near peripheral retina. The reason that neither Anstis and Cavanagh (1991) nor Mullen and Boulton (1992) were able to 
(a)

$$
\begin{aligned}
& - \text { Colour } \\
& - \text { - Luminance }
\end{aligned}
$$

SAL

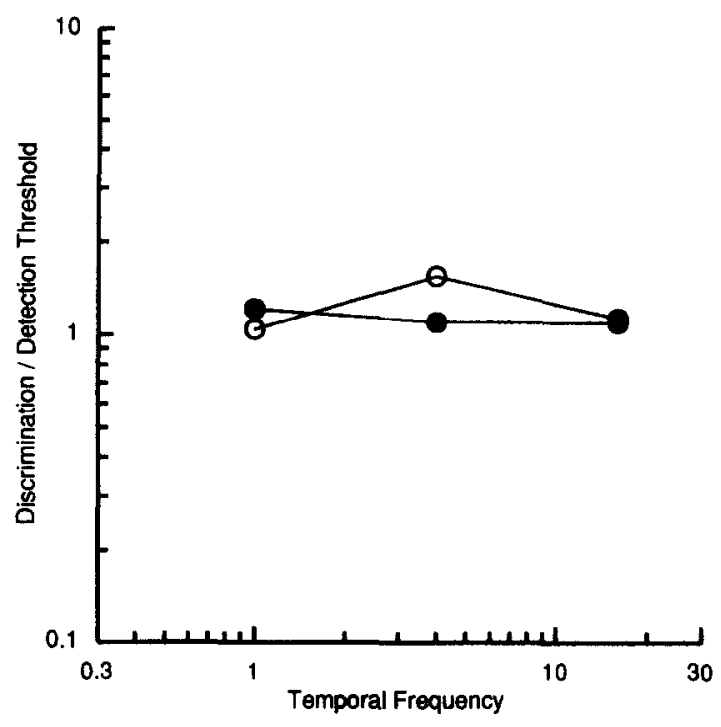

(b)

๑- Colour

Luminance

AMD

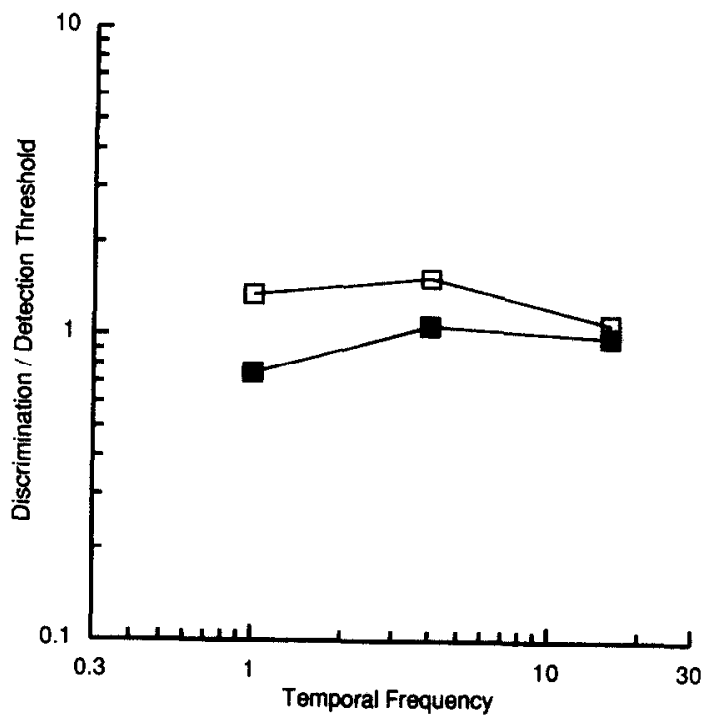

(c) $\mathrm{GBH}$
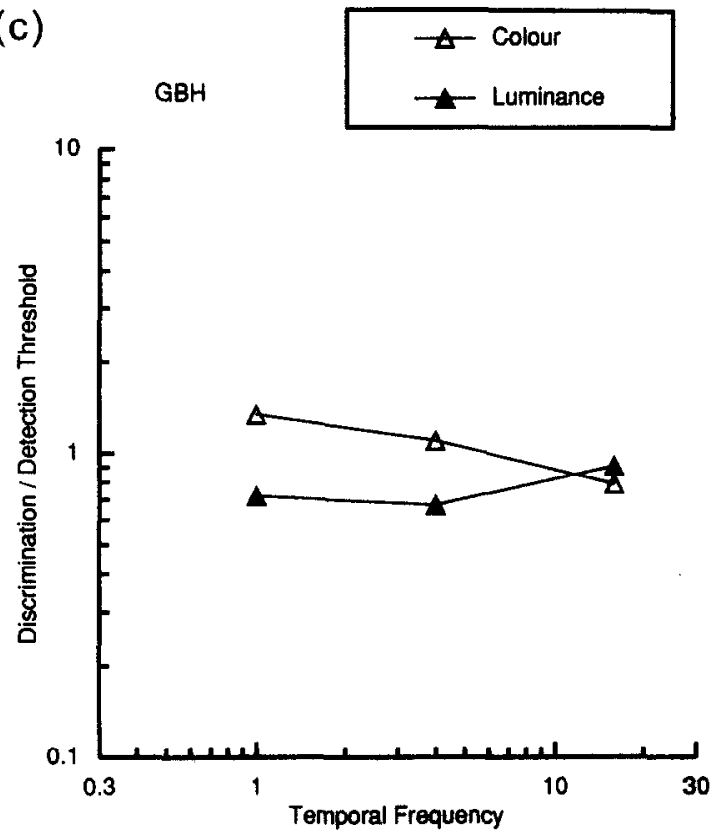

FIGURE 4. (a) The ratio of the direction-of-motion discrimination to detection "threshold" (the contrast corresponding to $75 \%$ correct judgements) as a function of temporal frequency $(\mathrm{Hz})$. Both axes are logarithmic. The solid symbols show the rcsults with luminance gratings, the open symbols those with colour gratings. The results are those of observer SAL. (b) As (a) but for observer AMD. (c) As (a) but for observer GBH.

replicate these results is that they presented their stimuli to the centre of the visual field.

\section{GENERAL DISCUSSION}

The most interesting aspect of these results is the effect of changing from a large, centrally fixated display to a smaller display viewed in the near periphery. With the large, centrally-fixated display for both colour and luminance gratings, direction of motion can be discriminated very close indeed to the detection threshold. This confirms the findings of Mullen and Boulton (1992) who found only a modest difference between detection and direction-of-motion discrimination thresholds using colour gratings, and suggests that Cavanagh and Anstis' (1991) subjective technique may have slightly over estimated the difference between detection and discrimination. Reducing the size of the stimulus and moving it to the near periphery appears to make it more difficult to detect the motion of colour gratings at contrasts close 
(a)

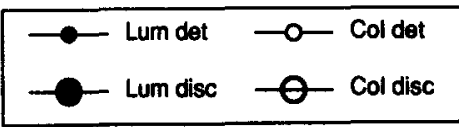

SAL

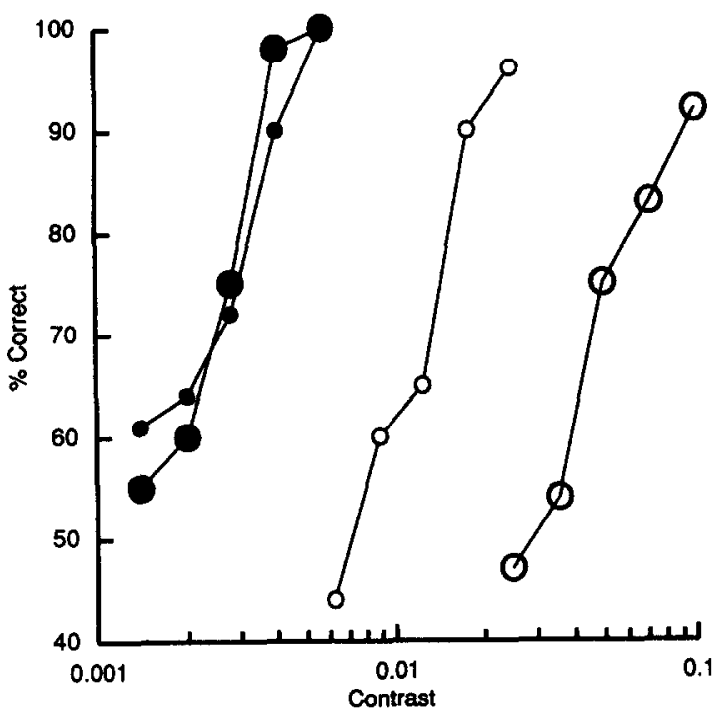

(b)

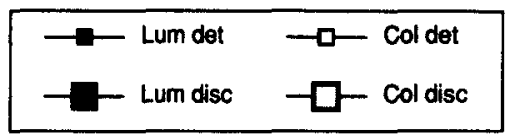

AMD

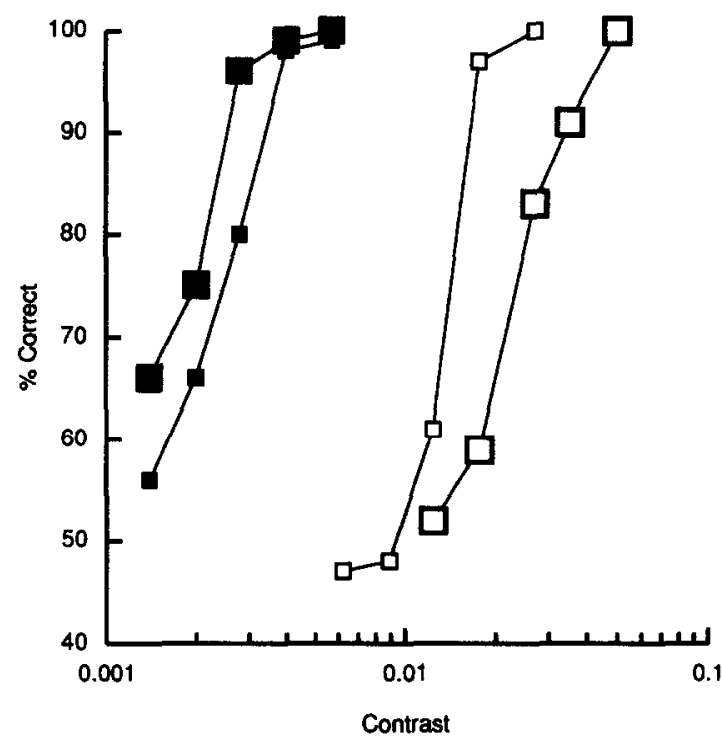

(c)

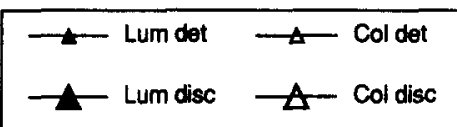

GBH

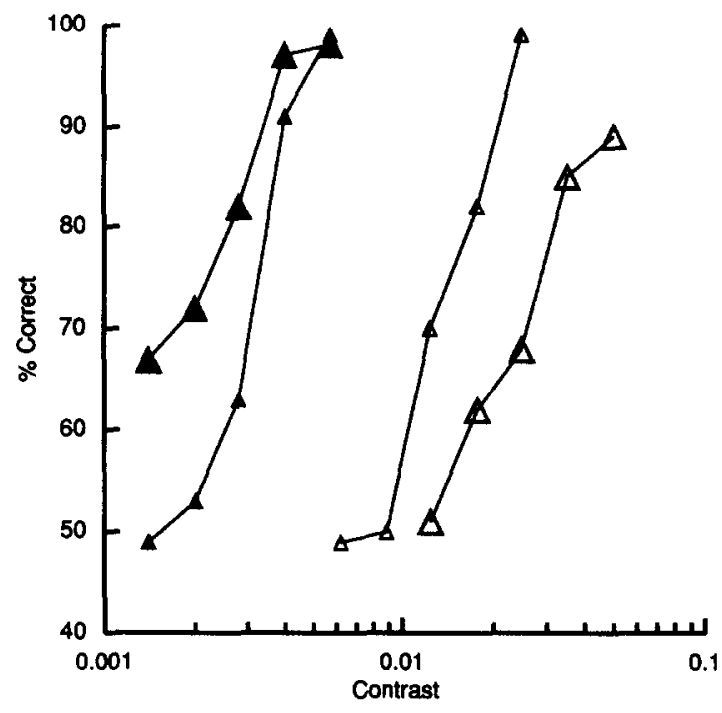

FIGURE 5. (a) The percentage correct direction-of-motion discrimination (large symbols) and the percentage of correct detection (small symbols) as functions of contrast. The patterns were presented within a circular window $2.5^{\circ}$ in diameter, centred $3.25^{\circ}$ to the left of the fixation mark. The solid symbols show the results with luminance gratings and the open symbols those with colour gratings. All the gratings moved with a temporal frequency of $4 \mathrm{~Hz}$ and each data point is based on 100 trials from observer SAL. (b) As (a) but for observer AMD. (c) As (a) but for observer GBH.

to detection threshold. This confirms the findings of Lindsey and Teller (1990), and reconciles their results with later work (Mullen \& Boulton, 1992). With centrally fixated stimuli, direction of motion can be discriminated close to detection threshold, for both colour and luminance stimuli, as reported by Cavanagh and Anstis (1991) and Mullen and Boulton (1992). When stimuli are presented in the near periphery the direction of motion of colour gratings cannot be discriminated until contrast is substantially above threshold (Lindsey \& Teller, 1990). 

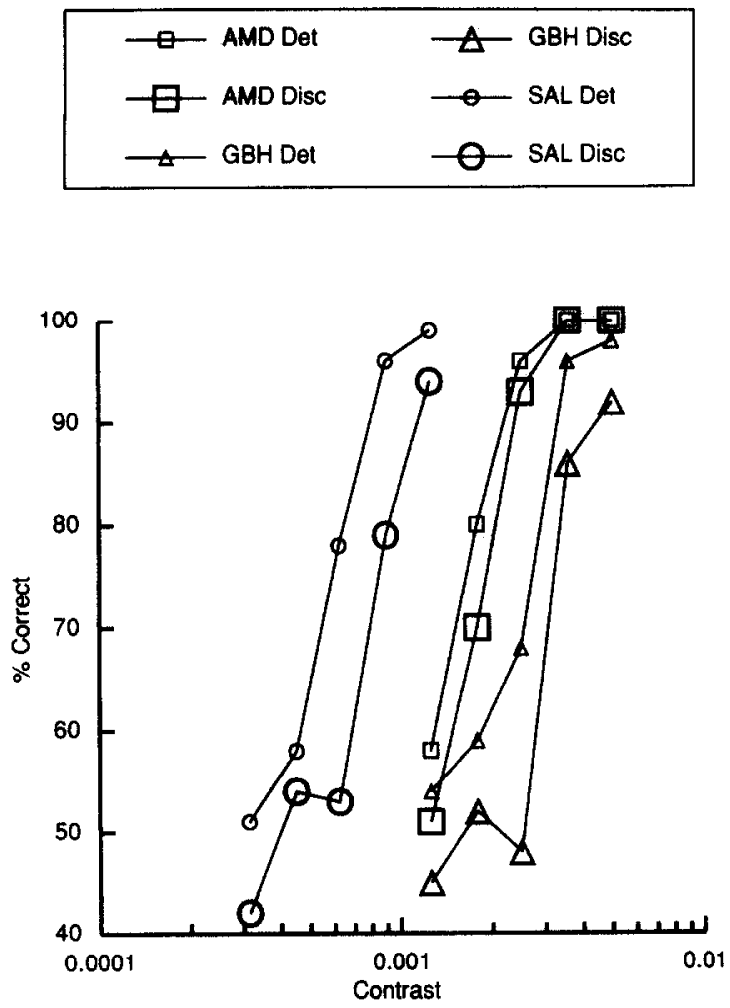

FIGURE 6. Detection (small symbols) and direction of motion discrimination (large symbols) for $1 \mathrm{c} / \mathrm{deg}$ colour gratings presented in a circular window $2.5 \mathrm{deg}$ in diameter and centred on the fixation point. Other details are as in Fig. 5. (Data for observer SAL have been displaced $0.6 \log$ units leftwards to avoid confusion.)

One interpretation of these results is in terms of different motion-detection systems whose existence has been proposed by others (Braddick, 1974, 1980). The "low-level" motion system is that associated with direction-selective filtering mechanisms which operate locally and produce the motion after-effect, but without extracting the location of image features. Chromatic signals can be expected not to contribute much to this system because it is specialized for extracting the motion of luminance signals, and so will not necessarily preserve the chromatic signature of its inputs from parvocellular neurones (Derrington \& Badcock, 1985a). The high-level motion system, on the other hand, functions by tracking the change in location of image features over time and may even represent a system that tracks shifts in attention (Cavanagh, 1991) and does not show a motion after-effect (Anstis, 1980), and may well be sensitive to spatial variations in colour.

Briefly, we suggest that at threshold and at the spatial frequency we use, the low-level system is sensitive to luminance gratings, but not to colour gratings, and that in central vision, but not in parafoveal vision, the high-level "feature-tracking" process is sensitive to chromatic signals (and probably also to luminance signals) at threshold. Thus the similarity between motion-discrimination thresholds and detection thresholds for colour gratings in central vision reflects the activity of the high-level process at threshold. The reason that this does not happen outside the fovea may simply be that the high-level process is much less effective there (Turano \&
Pantle, 1989). Outside the fovea the motion of chromatic gratings is not analysed until either the gratings become detectable by the low-level system, or exceed the raised threshold of the high-level system. The motion of luminance gratings can be analysed at threshold everywhere, because they activate the low-level system at threshold, as has already been suggested (Watson, Thompson, Murphy \& Nachmias, 1980). Our hypothesis about the role of the high-level motion system is speculative, but there are a number of results that support it, and it suggests some new experiments. However, before discussing them we will develop the main idea: that colour signals are processed by the low-level motion system, but not at threshold.

First, we consider the evidence that the colour signals are processed by the low-level motion system. In assessing the physiological results we provisionally identify the physiological substrate of the low-level motion system as the pathway from striate cortex to the middle temporal cortical area (MT), an area which has been identified as important in the analysis of motion since the discovery that the bulk of neurons there has strongly direction selective receptive fields (Dubner \& Zeki, 1971). Although the main pathways from V1 to area MT originate in layers which receive input from the magnocellular layers of the LGN [see Lennie, Trevarthen, Van Essen and Wässle (1990) for a review] there are two reasons for supposing that the parvocellular layers could also project to MT. First, adjacent cortical layers receive input from magnocellular and parvocellular laminae, and dendritic fields probably cross the boundary between the two laminae (Lennie et al., 1990), thus a parvocellular projection to the motion area is possible. Second, the signals of parvocellular neurons would be useful for the analysis of motion, particularly at high spatial frequencies and at high contrasts, so input from parvocellular laminae to the motion pathway is desirable. Thus it is no great surprise that direction selective cells in area MT give direction-selective responses to equiluminant stimuli of high chromatic contrast (Saito, Tanaka, Isono, Yasuda \& Mikami, 1989).

In psychophysics it is also difficult to define the low-level motion system unequivocally, however, we use two criteria: first, stimuli which are not detected by the low-level system do not give rise to a motion after-effect (Anstis, 1980; Derrington \& Badcock, 1985b). Thus we assume that stimuli which do give rise to a motion after-effect are detected by the "low-level" system. Second, the direction of motion of luminance patterns can be discriminated after very brief exposures $(10-20 \mathrm{msec})$, provided that the speed is high enough, whereas the direction of motion of patterns which consist of spatial variations in contrast requires an exposure about an order of magnitude longer (Derrington \& Henning, unpublished observations; Derrington, Badcock \& Henning, 1993). Thus a stimulus whose direction of motion can be discriminated in an exposure $<30 \mathrm{msec}$ can be assumed to be detected by the "lowlevel" motion system. 
Equiluminant colour gratings satisfy both these criteria, although they may not do so at contrasts close to the detection threshold. Equiluminant colour gratings give rise to a motion after-effect (Cavanagh \& Favreau, 1985; Derrington \& Badcock, 1985a; Mullen \& Baker, 1985), and the motion after-effects elicited by luminance gratings can be nulled by colour gratings and vice versa (Derrington \& Badcock, 1985a). The direction of motion of luminance gratings can be discriminated in exposures as short as $15 \mathrm{msec}$ at contrasts $0.5 \mathrm{log}$ units above threshold; whereas colour gratings require an exposure of $240 \mathrm{msec}$ at low contrasts, or a higher contrast in order to support direction-of-motion discrimination (Cropper \& Derrington, 1990): the direction of motion of colour gratings can be discriminated at exposure durations of $30 \mathrm{msec}$ at contrasts $1.5 \mathrm{log}$ units above threshold. The transition from slow to fast direction-of-motion discrimination for colour gratings takes place at about $1 \log$ unit above threshold (Cropper, 1992), suggesting that colour gratings cannot be processed by the low-level motion system until they are well above threshold. In preliminary experiments on motion after-effects we find that colour gratings do not elicit a motion after-effect at contrasts close to threshold, whereas it is well established that luminance gratings are extremely effective at eliciting after-effects at contrasts close to threshold (Keck, Palella \& Pantle, 1976). Thus it seems clear that colour signals do not gain access to the low-level motion system defined psychophysically at threshold; why should this be?

The most likely reason that colour signals do not activate the low-level motion system until they are well above threshold is that the colour signal and the motion signal are extracted independently of one another in different cortical areas (Zeki, 1978), and that the mechanism extracting the colour signal is more sensitive to chromatic contrast than the mechanism extracting the motion signal. This can be seen by considering what kind of processing is needed to construct colour-selective and motion-selective receptive fields from parvocellular neurons. The receptive fields of the commonest type of parvoccllular ncuron, named Type I by Wiesel and Hubel (1966), carry a duplex signal that combines luminance and chromatic information. They carry both a luminance signal that is band-pass in terms of its spatial frequency selectivity, and a chromatic signal that is low-pass (Derrington, Krauskopf \& Lennie, 1984). Thus further processing is required to extract either of these signals uncontaminated by the other. The detection threshold for colour gratings is likely to reflect the characteristics of the process which extracts the colour signal. However, any "low-level" processing of the signals from parvocellular neurons used to extract motion information probably occurs in parallel with, and separately from, the processing to extract the colour signal, and appears to be carried out in a way which does not lead to high sensitivity to chromatic contrast. Figure 7 illustrates why this might be so; it shows an example of minimal connection schemes: (a) to
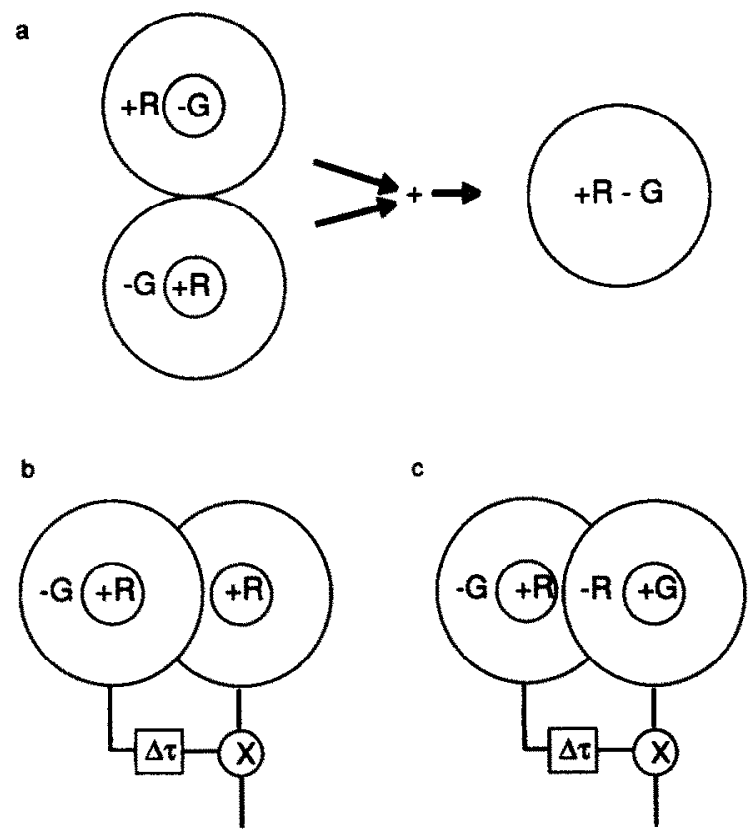

FIGURE 7. (a) A schematic receptive field organization which will generate a pure colour opponent receptive field by adding together the outputs of two spatially superimposed circular type I receptive fields of identical spatial profiles, but opposite response polarities. The input receptive fields show a combination of colour opponency (both receive excitatory inputs from $R$ cones and inhibitory inputs from $G$ cones), and spatial opponency (one has an excitatory centre and inhibitory surround, the other has an inhibitory centre and excitatory surround). The receptive field produced by summing these two superimposed receptive fields will be excited by red cones and inhibited by $\mathbf{G}$ cones (redrawn with permission from D'Zmura \& Lennie, 1986). (b) A schematic receptive field organization based on the model of Reichardt (1961) which combines the outputs of two spatially separated but otherwise identical circular $R-G$ colour opponent receptive fields to produce a receptive field which is selective for rightward motion of luminance or colour patterns. (c) A similar receptive field which has input receptive fields which are matched for their luminance sensitivity but not for their chromatic sensitivity. This receptive field will be selective for rightward motion of luminance patterns and for leftward motion of chromatic patterns (redrawn with permission from Derrington \& Badcock, 1985).

extract the colour signal [Fig. 7(a)] and (b) to extract a motion signal [Fig. 7(b)]. The hypothetical colour neuron in Fig. 7(a) sums the responses of two parvocellular neurons, and thus we would expect its output to exceed threshold if the summed activity of its two inputs exceeds threshold. It could well have a sensitivity to chromatic contrast which exceeds that of either of its two inputs. On the other hand, both of the minimal motion-detecting schemes have two inputs whose outputs are effectively multiplied to generate the motion signal, thus no output should be generated unless both the input stages generate suprathreshold signals. Thus we might expect the chromatic contrast required to support direction-of-motion discrimination to be above the thresholds of the two input neurons, and certainly higher than that required to support detection. The extent of the difference will depend on the relative degree of summation in colour receptive fields, and in the input stages of motion-detecting sub-units. Since both the spatial (Mullen, 1985) and the temporal (de Lange, 1958) 
sensitivity of colour detection mechanisms are low-pass we might expect a substantial degree of summation, and so a substantial enhancement in sensitivity for detection tasks over direction discrimination.

Furthermore, as Derrington and Badcock (1985a) point out, if the processing of parvocellular signals to extract motion is aimed at extracting a motion signal from luminance patterns we can expect a significant fraction of the motion-extracting sub-units to combine colour opponent units in incompatible ways, so that the detector signals motion in one direction for luminance patterns, and in the opposite direction for chromatic patterns. This is a special example of the general case that the different components of motion-detecting subunits should have identical colour selectivity for maximal motion sensitivity (Srinivasan, 1985). An example of an incompatible combination is shown in Fig. 7(c). The existence of a proportion of incompatible detectors of this type could well explain the weak and uncertain impression of motion that is obtained with equiluminant patterns, and the fact that it can be mimicked by adding spatial noise to luminance patterns (Troscianko \& Fahle, 1988).

\section{Conclusions and suggestions}

Our results are consistent with the hypothesis that high-level motion mechanisms, but not low-level motion mechanisms process colour signals at threshold. This hypothesis is being tested by studying motion aftereffects using colour gratings in foveal vision. Preliminary results suggest that colour gratings do not show a motion after-effect at chromatic contrasts close to threshold. Study of motion after-effects using chromatic gratings presented in parafoveal vision should show whether the process that detects their motion is highlevel or low-level.

\section{REFERENCES}

Anstis, S. M. (1980). The perception of apparent movement. Philesophical Transactions of the Royal Society of London B, 290, 153-168.

Braddick, O. J. (1974). A short-range process in apparent motion. Vision Research, 14, 519-527.

Braddick, O. J. (1980). Low-level and high-level processes in apparent motion. Philosophical Transactions of the Royal Society of London, 290, 137-151.

Cavanagh, P. (1991). No slowing for active motion perception at equiluminance. Investigative Ophthalmology and Visual Science, 32, 894.

Cavanagh, P. \& Anstis, S. (1991). The contribution of color to motion in normal and color-deficient observers. Vision Research, 31, $2109-2148$

Cavanagh, P. \& Favreau, O. E. (1985). Colour and luminance share a common motion pathway. Vision Research, 25, 1595-1601.

Cavanagh, P., Tyler, C. W. \& Favreau, O. E. (1984). Perceived velocity of moving chromatic gratings. Journal of the Optical Society of America, A1, 893-899.

Cropper, S. J. (1992). Motion discrimination: Different patterns, different detectors. PhD thesis, University of Neweastle upon Tyne, England.

Cropper, S. J. \& Derrington, A. M. (1990). The effects of contrast and duration on the discrimination of direction of motion in beats and chromatic gratings. Perception, 19, 353.
Derrington, A. M. \& Badcock, D. R. (1985a). The low level motion system has both chromatic and luminance inputs. Vision Research, $25,1879-1884$.

Derrington, A. M. \& Badcock, D. R. (1985b). Separate detectors for simple and complex grating patterns? Vision Research, 25, $1869-1878$.

Derrington, A. M. \& Henning, G. B. (1987). Errors in direction-of motion discrimination with complex stimuli. Vision Research, 27, 61-75.

Derrington, A. M., Badcock, D. R. \& Henning, G. B. (1993) Discriminating the direction of second-order motion at short stimulus durations. Vision Research. Submitted.

Derrington, A. M., Krauskopf, J. \& Lennie, P. (1984). Chromatic mechanisms in lateral geniculate nucleus of macaque. Journal of Physiology, 357, 241-265.

Dubner, R. \& Zeki, S. (1971). Response properties and receptive fields in an anatomically defined area of the superior temporal sulcus in the monkey. Brain Research, 35, 528-532.

D'Zmura, M. \& Lennie, P. (1986). Mechanisms of colour constancy. Journal of the Optical Society of America, A3, 1662-1672.

Keck, M. J., Palella, T. D. \& Pantle, A. (1976). Motion aftereffect as a function of the contrast of sinusoidal gratings. Vision Research, 16, 187-191.

Kelly, D. H. (1983). Spatiotemporal variation of chromatic and achromatic contrast thresholds. Journal of the Optical Society of America, 73, 742-750.

Krauskopf, J. \& Farell, B. (1990). Influence of colour on the perception of coherent motion. Nature, 348, 328-331.

de Lange, H. (1958). Research into the dynamic nature of the human fovea-cortex systems with intermittent and modulated light. 11. Phase shift in brightness and delay in color perception. Journal of the Optical Society of America, 48, 784-789.

Lennie, P. \& D'7mura, M. (1988). Mechanisms of color vision. CRC Critical Reviews in Neurobiology, 3, 333-400.

Lennie, P., Trevarthen, C., Van Essen, D. \& Wässle, H. (1990). Parallel processing of visual information. In Spillman, L. \& Werner, J. S (Eds), Visual perception the neurophysiological foundations (pp. 102-128). San Diego, Calif: Academic Press.

Lindsey, D. T. \& Teller, D. Y. (1990). Motion at isoluminance: Discrimination/detection ratios for moving isoluminant gratings. Vision Research, 30, 1751-1761.

Mullen, K. T. (1985). The contrast sensitivity of human colour vision to red-green and blue-yellow chromatic gratings. Journal of Physiology, 359, 38I-400.

Mullen, K. T. \& Baker, C. L. (1985). A motion aftereffect from an isoluminant stimulus. Vision Research, 25, 685-688

Mullen, K. T. \& Boulton, J. C. (1992). Absence of smooth motion perception in color vision. Vision Research, 32, 483-488.

Pelli, D. G. \& Zhang, L. (1991). Accurate control of contrast on microcomputer displays. Vision Research, 31, 1337-1350.

Ramachandran, V. S. \& Gregory, R. L. (1978). Does colour provide an input to human motion perception? Nature, 275, 55-56.

Reichardt, W. (1961). Autocorrelation, a principle for the evaluation of sensory information by the central nervous system. In Rosenblith, W. A. (Ed.), Sensory communication (pp. 303-317). New York: Wiley.

Robson, J. G. (1966). Spatial and temporal contrast sensitivity functions of the visual system. Journal of the Optical Society of America. $56,1141-1142$.

Saito, H., Tanaka, K., Isono, H., Yasuda, M. \& Mikami, A. (1989) Directionally selective response of cells in the middle temporal area (MT) of the macaque monkey to the movement of equiluminous opponent colour stimuli. Experimental Brain Research, 75, 1-14 Schade, O. H. (1956). Optical and photoelectric analog of the eye Journal of the Optical Society of America, 46, 721-739.

Smith, V. C. \& Pokorny, J. (1975). Spectral sensitivity of the foveal cone photopigments between 400 and $500 \mathrm{~nm}$. Vision Research, 15, $161-171$.

Srinivasan, M. V. (1985). Shouldn't directional movement detection necessarily be "colour-blind"? Vision Research, 25, 997-1000.

Stromeyer III, C. F., Cole, G. R. \& Kronauer, R. E. (1987). Chromatic suppression of cone inputs to the luminance flicker mechanism. Vision Research, 27, 1113-1137. 
Stromeyer III, C. F., Eskew, R. T. J. \& Kronauer, R. E. (1990). The most sensitive motion detectors in humans are spectrallyopponent. Investigative Ophthalmology and Visual Science, 31, 240.

Troscianko, T. \& Fahle, M. (1988). Why do isoluminant stimuli appear slower? Journal of the Optical Society of America, AS, 871-880.

Turano, K. \& Pantle, A. (1989). On the mechanism that encodes the movement of contrast variation: Velocity discrimination. Vision Research, 29, 207-221.

Varner, D., Jameson, D. \& Hurvich, L. M. (1984). Temporal sensitivities related to color theory. Journal of the Optical Society of America, A1, 474-481.

Watson, A. B., Thompson, P. G., Murphy, B. J. \& Nachmias, J.
(1980). Summation and discrimination of gratings moving in opposite directions. Vision Reseurch, 20, 341-347.

Wiesel, T. N. \& Hubel, D. H. (1966). Spatial and chromatic interactions in the lateral geniculate body of the rhesus monkey. Journal of Neurophysiology, 29, 1115-1156.

Zeki, S. M. (1978). Functional specialization in the visual cortex of the rhesus monkey. Nature, 274, 423-428.

Acknowledgements - We are grateful to the SERC for financial support to AMD through grant GR/G/730, to Simon Cropper for letting us use his computer programmes, and to Ms Sandra Loaiza for observing and technical assistance. 